\title{
Methods and Results in Characterizing Electronic Stethoscopes
}

\author{
RL Watrous, DM Grove, DL Bowen* \\ Zargis Medical Corporation, Princeton, NJ USA \\ ${ }^{*}$ RH Lyon Corp, Cambridge, MA USA
}

\begin{abstract}
Two methods for characterizing the acoustical properties of electronic stethoscopes are reported. The first method measures the frequency response of the stethoscope using a fluid-filled rubber ball that is excited by a mechanical shaker driven by broadband noise. The acoustic impedance of this phantom is designed to be similar to that of human body tissue. The second method measures the acoustic impedance of the stethoscopes using a standard acoustic impedance tube measurement system. The frequency response and impedance results for six commercially available electronic stethoscopes are reported. It is shown that the stethoscope responses vary considerably as a function of frequency, showing peaks and nulls of as much as $20 \mathrm{~dB}$ in the frequency range of $5 \mathrm{~Hz}$ to $3 \mathrm{kHz}$ and that the stethoscope responses differ considerably from each other.
\end{abstract}

\section{Introduction}

The development of computer-based diagnostic decision support systems for the analysis and interpretation of heart and lung sounds depends critically on the electro-acoustic properties of the sensors, typically embodied in electronic stethoscopes, used to acquire the signals of interest. There are, however, no existing acoustical standards for characterizing electronic stethoscopes.

A lack of standard measurement procedure puts into doubt one manufacturer's claim over another's in terms of device sensitivity. Listening tests to choose an electronic stethoscope do not provide an objective measure of device performance. Moreover, without knowledge of some absolute transfer function, it becomes difficult to change stethoscopes for diagnostic decision support signal processing.

This paper presents two methods for characterizing electronic stethoscopes: i) sensitivity based upon freemotion acceleration using a phantom and ii) acoustical impedance of chest piece coupler to skin. Several commercially available electronic stethoscopes are tested and compared. The results suggest that these methods are useful for characterizing electronic stethoscopes for instrumentation purposes.

\section{Experiment setup}

There are no existing standards for characterizing electronic stethoscopes. Two competing methods have emerged for measuring the frequency response of acoustic stethoscopes that can be carried over to the electronic version. One method uses air coupling [1,2] while the second uses a phantom [3,4].

The first method uses an air coupler to transmit frequencies to the chest piece and measures the input pressure to the chest piece. Another air coupler is attached to the earpieces to measure the pressure at this location. The electronic stethoscope requires measuring output voltage from the chest piece to the input pressure from the loudspeaker attached to the other end of the chest piece coupler.

Difficulties with this method lie in the assumption of infinite impedance by the air coupler. This testing method does not show the results for the stethoscope in normal use. There will be a shift in the frequency response due to the compliance that is not taken into account by this method [5]. Another issue involves maintaining a sealed coupling over various electronic stethoscopes since there are no standard shapes/sizes.

The second method places the stethoscope on a phantom to approximate the vibrating condition of the chest. The phantom is designed to approximate the impedance of the chest wall. A vibrating source is typically placed inside the source. The output stethoscope voltage to the vibration measurement is recorded as the frequency response. This second method is used for the frequency response measurements reported on in this work.

Another issue is in matching the acoustic impedance of the stethoscope to that of the chest wall. If this condition is met, good sound transmission is assured at the transducer. While many manufacturers give some indication of frequency response, there are no measures of acoustic impedance. We propose to measure this parameter as well. 


\subsection{Frequency response setup}

A $16.5 \mathrm{~cm}$ diameter water-filled polymer ball is used for the phantom. Each stethoscope is held against the ball, by hand, with enough pressure to simulate an actual heart recording. A Labworks ET-132 shaker excites the outside of the sphere. This excitation method is in accordance with [6,7]. An Endevco 2-gm accelerometer separately records the undisturbed surface acceleration without the stethoscope in place, at a number of different points within the contact area. Figure 1 shows a photograph of the recording setup, including the stethoscope placement.

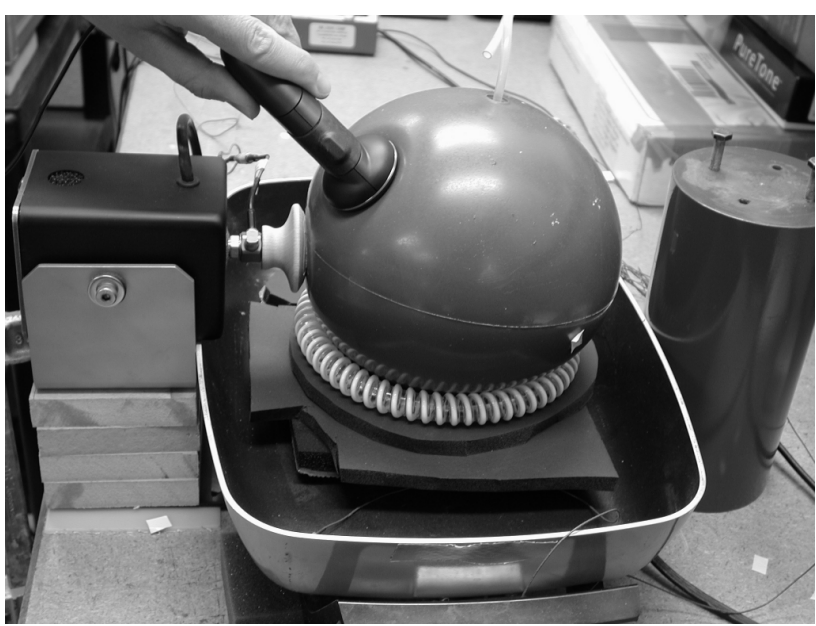

Figure 1. Frequency response test setup, including shaker and stethoscope placement.

The sensitivity of the stethoscope is determined as a ratio of the stethoscope output voltage from the chest piece to the average free-surface acceleration obtained from the accelerometer measurements. The shaker is driven with shaped pseudo-random noise and the sensitivity computed over a frequency range of $20 \mathrm{~Hz}-5$ $\mathrm{kHz}$, with 64 averages.

The background noise input to the stethoscope, while placed on the phantom, is measured with the shaker off and amplification equipment on. The noise floor remained $10 \mathrm{~dB}$ below the signal above approximately 30 $\mathrm{Hz}$.

A separate measurement is used for low frequency sensitivity measurements from $5-55 \mathrm{~Hz}$. For this frequency range, the shaker drives the phantom over a smaller frequency range, with a different noise shaping applied.

A coherence function from the shaker input to the accelerometer output was monitored to set the initial noise shaping. The results were judged to be adequate for these measurements.

\subsection{Acoustic impedance setup}

To allow for maximum sound transmission, and maximum force input to the stethoscope, the mechanical impedance for both the chest piece coupler and chest wall should be similar. The normalized specific acoustic impedance of the coupler, $\varsigma=z / \rho c$, is measured, where $\rho c$ is the air impedance, 407 rayls. The mechanical impedance is then

$$
Z_{\text {mech }}=\varsigma \rho c A
$$

where $\mathrm{A}$ is the cross-sectional area of the contacting surface.

The acoustic impedance is measured using a twomicrophone method with a standard impedance tube [8]. The sample is mounted on the end of a stainless steel tube of $0.001 \mathrm{~m}^{2}$ cross-sectional area. A direct radiator loudspeaker at the opposite end of the tube transmits white noise between $50 \mathrm{~Hz}-5 \mathrm{kHz}$. The complex transfer function, $\boldsymbol{H}$, is recorded from two $1 / 4^{\prime \prime}$ PCB microphones spaced $3.18 \mathrm{~cm}$ apart. Spectronics software computes the normalized specific acoustic impedance from the transfer function.

The transfer function is the ratio of the complex crossspectrum to the input autopower spectrum. The normalized specific impedance, $\zeta$, is calculated from the complex reflection coefficient $\boldsymbol{R}$ as $(1+\boldsymbol{R}) /(1-\boldsymbol{R})$. The reflection coefficient is computed from the transfer function as

$$
\boldsymbol{R}=\frac{\boldsymbol{H}-e^{-j k s}}{e^{j k s}-\boldsymbol{H}} e^{j 2 k(l+s)}
$$

where $k$ is the wavenumber, $s$ is the microphone spacing, and $l$ is the distance from the test sample to the nearest microphone.

\subsection{Electronic stethoscopes tested}

Table 1 shows the 6 stethoscopes tested, along with some of their distinguishing traits. The diaphragms for stethoscopes $\mathrm{B}$ and $\mathrm{F}$ are of similar very stiff plastic material. Stethoscope $\mathrm{C}$ has a very compliant soft cover for the diaphragm. The coupler for D forms an outer seal with a smaller interior contacting surface. Stethoscope A has a plastic coupler to the skin.

Table 1. Electronic stethoscopes tested.

\begin{tabular}{cll}
\hline Stethoscope & Transducer Type & Type Skin Coupler \\
\hline A & Force Gage & Diaphragm \\
B & Electret & Diaphragm \\
C & Electret & Diaphragm/Bell \\
D & Force Gage & Diaphragm \\
E & Electret & Shallow Bell \\
F & Electret & Diaphragm \\
\hline
\end{tabular}




\section{Results}

The results for sensitivity relative to free-motion acceleration and normal specific acoustic impedance are presented in this section.

\subsection{Frequency response comparisons}

The frequency response for all stethoscopes relative to free-motion acceleration is shown in figures 2 and 3 . None of the electronic stethoscopes tested exhibit a flat response. Each device shows multiple peaks and nulls in the response. The resonance frequencies vary with device. Only devices $\mathrm{D}$ and $\mathrm{F}$ have similar sensitivities in terms of magnitude and resonance location.

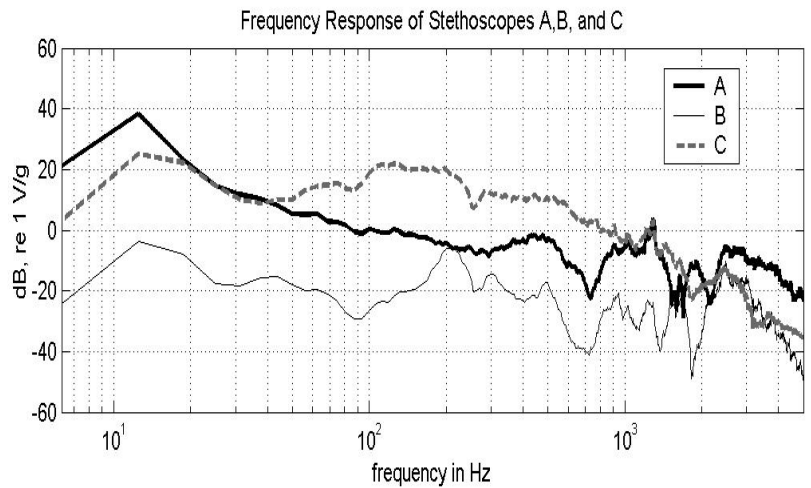

Figure 2. Sensitivity relative to free-motion acceleration for devices A, B, and C.

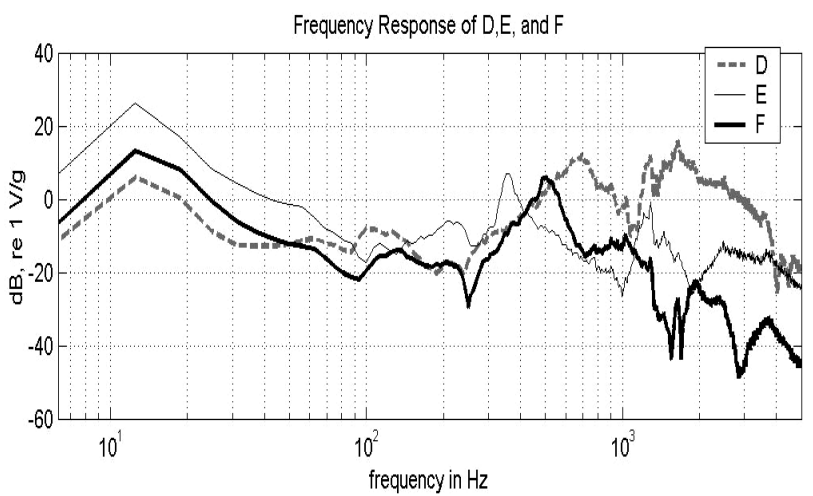

Figure 3. Sensitivity relative to free-motion acceleration for devices $\mathrm{D}, \mathrm{E}$, and $\mathrm{F}$.

For all of these devices, the response can be separated into 4 regions. Region I is the low frequency range. All devices follow the same behavior of a resonance at 12.5 $\mathrm{Hz}$ and decreasing response. Region II is the mid frequency range. In this region the response is fairly flat with marginal large resonances. Region III is the high frequency region characterized by a significant number of peaks and nulls. Region IV is where significant frequency rolloff occurs. Table 2 summarizes the frequency regions for each stethoscope.

Table 2. Summary of frequency regions (in $\mathrm{Hz}$ ) for each stethoscope.

\begin{tabular}{cllll}
\hline Steth & I & II & III & IV \\
\hline A & $0-100$ & $100-600$ & $700-3000$ & $>3.5 \mathrm{k}$ \\
B & $0-90$ & $90-550$ & $600-3000$ & $>3.0 \mathrm{k}$ \\
C & $0-90$ & $90-600$ & ------ & $>600$ \\
D & $0-90$ & $90-250$ & $250-3500$ & $>3.5 \mathrm{k}$ \\
E & $0-100$ & $100-250$ & $250-3500$ & $>3.5 \mathrm{k}$ \\
F & $0-100$ & $100-250$ & $250-3500$ & $>3.5 \mathrm{k}$ \\
\hline
\end{tabular}

Region I: All devices in this region exhibit similar behavior. Device A has the highest sensitivity in this region. Device $\mathrm{C}$ has next highest sensitivity and the least amount of rolloff compared to all other devices.

Region II: In this region, devices A-C have the largest frequency range consisting of a fairly flat response with devices $\mathrm{A}$ and $\mathrm{C}$ having the least variations. Device $\mathrm{C}$ exhibits the largest sensitivity in this region.

Region III: This region is characterized by a general decrease in sensitivity and multiple peaks and nulls. Device D has the highest sensitivity in this region. It is also the only device to emphasize this high frequency region over its low frequency response.

Region IV: A rapid decrease in sensitivity with increasing frequency occurs in this region. Device $\mathrm{C}$ does not exhibit the same pattern of multiple peaks above region II. Instead this device has a gradual decrease in sensitivity similar to the characteristics of region IV.

A comparison of sensitivity was examined with light and normal pressure on stethoscope A only. No significant difference in resonance or sensitivity magnitude was found.

While all stethoscopes have volume adjustability for the earpieces, not all of them can adjust the amplitude of the electronic signal from the chest piece. For any stethoscope that had a variable gain electronic signal, the volume was set at its maximum level for the sensitivity measurements.

\subsection{Acoustic impedance comparisons}

The phantom impedance was compared against skin impedance and found to have similar characteristics. The specific acoustic impedance of skin falls in the region of $50 \mathrm{~Hz}$ to $900 \mathrm{~Hz}$. There are several resonances in this region. This is shown in figure 4 . The phantom has similar impedance as skin but with approximately double the magnitude and higher Q.

The impedance for all of the stethoscopes tested fall into classes based upon resonances and impedance magnitude. The results are shown in figure 5. 


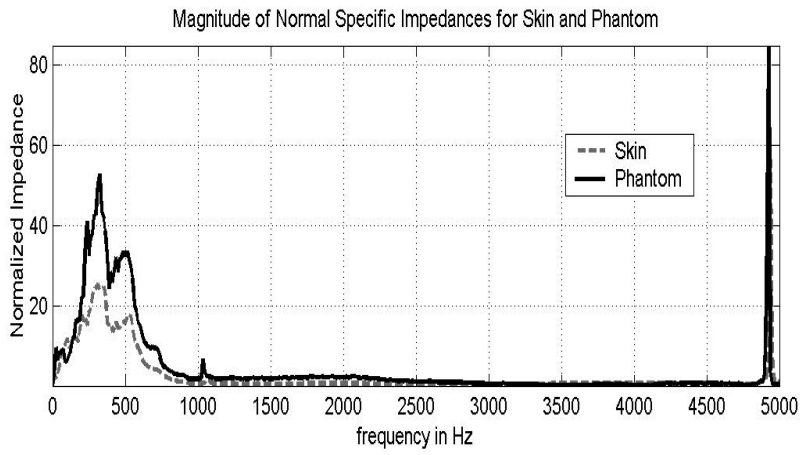

Figure 4. Magnitude of normalized specific acoustic impedance for phantom and skin.
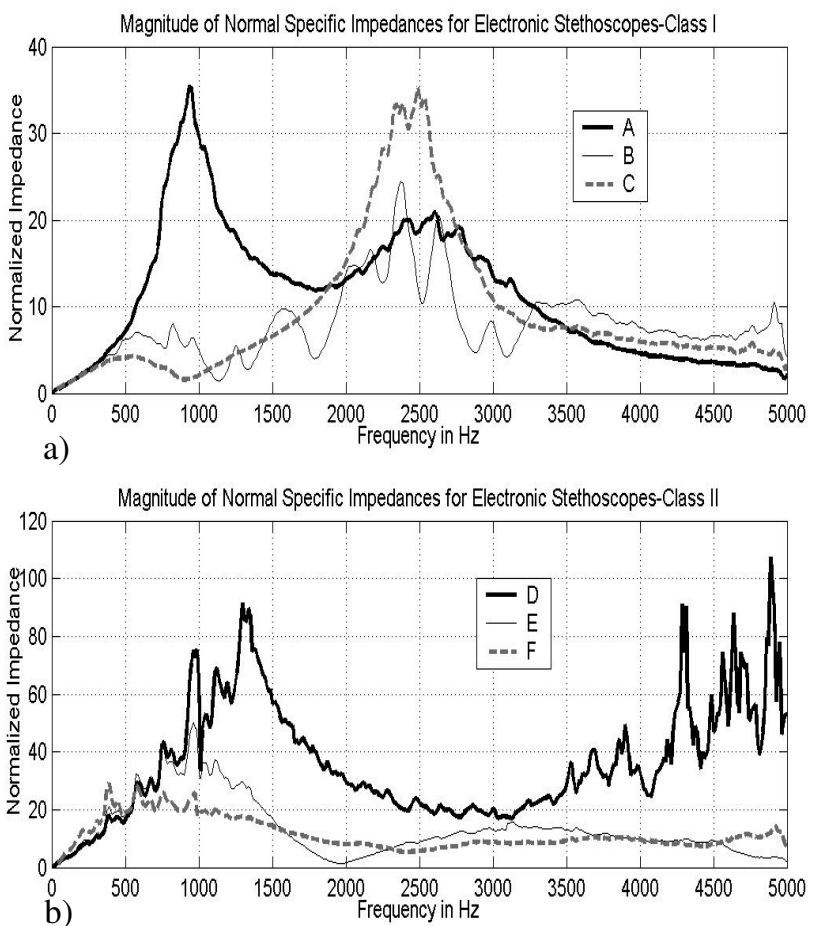

Figure 5. Magnitude of normal specific acoustic impedance for a) Class I and b) Class II chest pieces.

Class I stethoscopes all have a broad resonance around $2.5 \mathrm{kHz}$. Both $\mathrm{B}$ and $\mathrm{C}$ have only a single resonance. Device A has an additional resonance at $900 \mathrm{~Hz}$ but the magnitude is similar to the other devices.

Class II stethoscopes all contain a resonance between 500-1500 Hz. Device F has the lowest impedance and also the resonance closest to that of skin. Device D has the largest magnitude over the entire frequency range.

\section{Conclusions}

There is a wide variation in the acoustical properties of commercially available stethoscopes. Each stethoscope exhibits resonances/anti-resonances that arise from the choice of sensor and mechanical design. Many of these peaks are of large variation in the same region as murmurs. No stethoscope tested has a flat frequency response, which is a more appropriate response for computational processing of the acoustical signal. None of the devices tested has an impedance closely matching that of skin tissue.

There are no standards for measuring and comparing the acoustical properties of electronic stethoscopes. The methods described provide a means for characterizing and comparing electronic stethoscopes in a manner consistent with their use. The impedance tube method can be used to develop a chest piece that approximates human tissue impedance. With the advent of processing techniques utilizing an electronic stethoscope, it behooves us to develop a standard measurement practice.

\section{Acknowledgements}

We would like to thank the technical staff at RH Lyon Corp for their help in carrying out the experiments.

\section{References}

[1] Ertel PY, Lawrence M, Song W. How to test stethoscopes. Med Res Eng 1969;8:7-17.

[2] Abella M, Formolo J, Penney DG. Comparison of the acoustic properties of six popular stethoscopes. J Acoust Soc Am 1992;91:2224-2228.

[3] Padmanabhan V, Semmlow JL, Welkowitz W. Accelerometer type cardiac transducer for detection of lowlevel heart sounds. IEEE Trans on Biomedical Eng 1993;40:21-28.

[4] Royston TJ, Zhang X. Modeling sound transmission through the pulmonary system and chest with application to diagnosis of a collapsed lung. J Acoust Soc Am 2002;111:1931-1946.

[5] Latimer KE. The calibration of stethoscopes and phonocardiographic microphones. Bibl Cardiol 1975;33:8790, 1975.

[6] Fredberg JJ. Turbulent pseudo-sound production in atherosclerotic arteries [dissertation]. Cambridge (MA): Massachusetts Institute of Technology; 1974.

[7] Kamm RD. A study of external pneumatic compression for the prevention of deep venous thrombosis [dissertation]. Cambridge (MA): Massachusetts Institute of Technology; 1977.

[8] Standard test method for impedance and absorption of acoustical materials using a tube, two microphones, and a digital frequency analysis system. ASTM E1050-98. West Conshohocken (PA): American Society for Testing and Materials; 1998.

Address for correspondence.

D. M. Grove

755 College Road East

Princeton, NJ 08540

dgrove@zargis.com 